\title{
Delayed Post COVID-19 Bronchial Artery Pseudoaneurysm Treated with Embolisation: Letter to the Editor
}

\author{
Simon A. Braithwaite ${ }^{1}$ (D) Daniel J. Kearns ${ }^{1} \cdot$ Eric Douglas $^{1} \cdot$ Rachel K. Hoyles $^{1}$ • \\ Charles R. Tapping ${ }^{1}$
}

Received: 12 March 2021 / Accepted: 24 May 2021/Published online: 9 June 2021

(C) Springer Science+Business Media, LLC, part of Springer Nature and the Cardiovascular and Interventional Radiological Society of Europe (CIRSE) 2021

\section{Dear Editor,}

The authors present a case, study of a patient with delayed massive haemoptysis from a pseudoaneurysm believed to have formed secondary to coronavirus disease 2019 (COVID-19) who was successfully treated with bronchial artery embolisation (BAE).

A 53-year-old man, with a background of asthma, high body mass index and who has never smoked, presented initially in March of 2019 with COVID-19. Following the initial admission, the patient suffered with chronic fatigue ('long COVID') at home but was otherwise well. Eight months later he developed sudden onset massive haemoptysis with up to $600 \mathrm{ml}$ of fresh red blood being expectorated in each episode.

Investigations to identify the source and aetiology of bleeding were initially inconclusive. Rigid bronchoscopy demonstrated blood in the right lower lobe but no bleeding source was identified. All serological testing for a cause was negative. All three mucosal SARS-CoV-2 swabs undertaken during this admission were negative. No causative organisms were identified on microscopy or grown on culture.

Sequential computed tomography (CT) scans demonstrated progressive right lower lobe consolidation, ground glass opacification and endobronchial opacification, consistent with progressive pulmonary haemorrhage. No pulmonary emboli were identified. The bronchial artery anatomy was demonstrated but the vessels were not significantly hypertrophied. There was a small rounded

Simon A. Braithwaite

simon.braithwaite1@nhs.net

Oxford University Hospitals Trust, Oxford, UK arterial enhancement adjacent to the right lower lobe bronchus continuous with the bronchial arteries, felt to be a small pseudoaneurysm (Fig. 1) which was not present on the previous admission, eight months before.

The patient continued to have further haemoptysis during the admission despite medical management and on day 4 the decision was made to attempt bronchial artery embolisation, which other authors have described in the acute phase [1]. Embolisation was performed via left common femoral percutaneous 5-French access. The bronchial artery origin was cannulated with 5-french Berenstein catheter and a 2.4-French ProGreat microcatheter (Terumo, Tokyo, Japan) was advanced over Fathom-16 guidewire (Boston Scientific, Marlborough, MT, USA) into the combined right middle and lower lobe

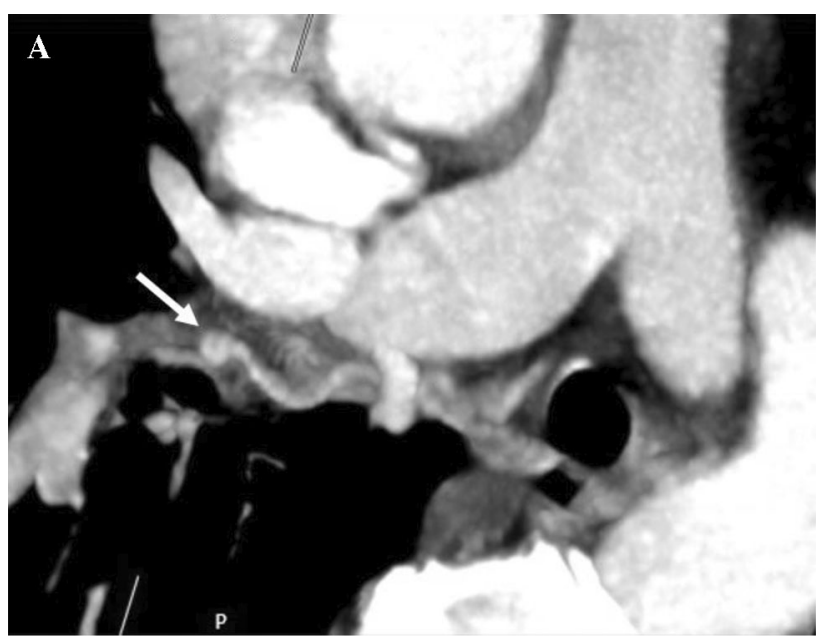

Fig. 1 Maximum intensity projection multiplanar reconstruction from $\mathrm{CT}$ angiogram showing the pseudoaneurysm in a right lower lobe bronchial artery (white arrow) 
Fig. 2 Digital subtraction angiography acquired in left anterior oblique projection preembolisation A showing the pseudoaneurysm (black arrow) and post-embolisation B showing no filling of abnormal artery (black arrow) and contrast refluxing into adjacent middle lobe arteries (white arrow)
A

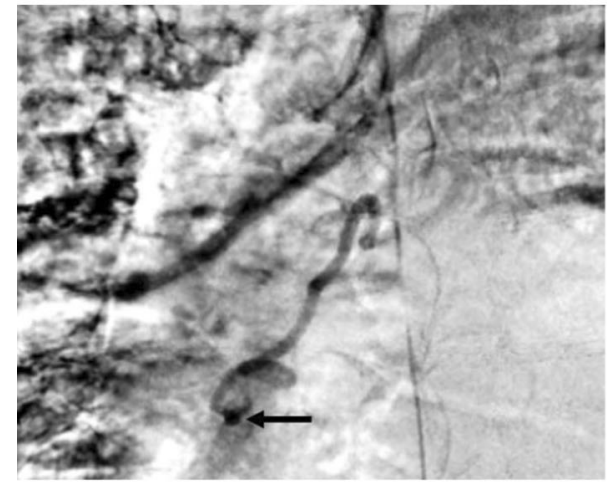

B

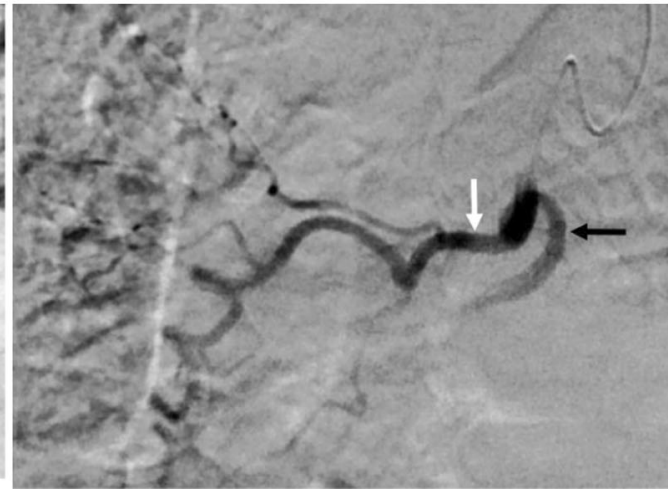

bronchial artery origin. The right lower lobe and middle lobe arteries demonstrated abnormal arterial and parenchymal enhancement with the right lower lobe pseudoaneurysm demonstrated (Fig. 2). These arteries were embolised using 500-700um Embospheres (Merit Medical, South Jordan, UT, USA). Particles were selected as embolic of choice, to preserve future embolisation access options as it was unclear whether future embolisation might be required, akin to patients with other lung disease [2].

In the two days following the procedure the patient expectorated increasingly old blood, following this there was no further haemoptysis and he showed gradual clinical improvement and was discharged 5 days after the embolisation. A repeat CT showed improvement in the consolidation of the right lung base.

The authors believe that this patient developed a bronchial artery pseudoaneurysm secondary to SARS-CoV-2 infection, which subsequently bled and presented as a delayed consequence of COVID-19. Although the relationship is not conclusive, other research such as Ackermann et al. [3] showed diffuse alveolar damage associated with both thrombotic and haemorrhagic lesions: severe endothelial injury, alveolar capillary microthrombi, and increased angiogenesis in the lungs of deceased COVID-19 patients. Lee et al. [4] described a pulmonary artery pseudoaneurysm in the context of H1N1 influenza viral pneumonia, and Savić et al. [5] described a case of a ruptured cerebral pseudoaneurysm which they believe developed in the context of COVID-19. The mechanism of haemoptysis remains unclear; however, the authors suggest a possible link between COVID-19 and pseudoaneurysm formation.
The authors suggest that bronchial artery embolisation is a feasible and successful management strategy for patients who present with haemoptysis and previous COVID-19. Bronchial artery pseudoaneurysms should be considered and pre-procedure planning with $\mathrm{CT}$ angiography is recommended.

\section{Declarations}

Conflict of interest The author declares that they have no conflict of interest.

\section{References}

1. Barral M, Sirol M, El Hajjam M, Zhang N, Petit A, Cornelis FH. Bronchial artery embolization performed in COVID-19 patients: tolerance and outcomes. Cardiovasc Intervent Radiol. 2020;43(12):1949-51.

2. Panda A, Bhalla AS, Goyal A. Bronchial artery embolization in hemoptysis: a systematic review. Diagn Interv Radiol. 2017;23(4):307-17. https://doi.org/10.5152/dir.2017.16454.

3. Ackermann M, Verleden SE, Kuehnel M, Haverich A, Welte T, Laenger F, et al. Pulmonary vascular endothelialitis, thrombosis, and angiogenesis in Covid-19. N Engl J Med. 2020;383(2):120-8.

4. Lee JC, Walters DL, Slaughter RE. Angioembolisation of pulmonary artery pseudoaneurysm arising in $\mathrm{H} 1 \mathrm{~N} 1$ influenza viral pneumonia. Hear Lung Circ [Internet]. 2011;20(9):599-601. https://doi.org/10.1016/j.hlc.2011.01.012.

5. Savić D, Alsheikh TM, Alhaj AK, Lazovic L, Alsarraf L, Bosnjakovic P, et al. Ruptured cerebral pseudoaneurysm in an adolescent as an early onset of COVID-19 infection: case report. Acta Neurochir (Wien). 2020;162(11):2725-9.

Publisher's Note Springer Nature remains neutral with regard to jurisdictional claims in published maps and institutional affiliations. 